\title{
Two-layer Scheduling Scheme for Pump Stations*
}

\author{
Cong Cong Sun ${ }^{1}$, Vicenç Puig $^{1}$ and Gabriela Cembrano ${ }^{1,2}$
}

\begin{abstract}
In this paper, a two-layer scheduling scheme for pump stations in a water distribution network has been proposed. The upper layer, which works in one-hour sampling time, uses Model Predictive Control (MPC) to produce continuous flow set-points for the lower layer. While in the lower layer, a scheduling algorithm has been used to translate the continuous flow set-points to a discrete (ON-OFF) control operation sequence of the pump stations with the constraints that pump stations should draw the same amount of water as the continuous flow set-points provided by the upper layer. The tuning parameters of such algorithm are the lower layer control sampling period and the number of parallel pumps in the pump station. The proposed method has been tested in the Richmond case study.
\end{abstract}

\section{INTRODUCTION}

In conventional water distribution systems, pumping water comprises the major fraction of the total energy budget. In practice, the operation of a pump station is simply a set of rules or a schedule that indicates when a particular pump or group of pumps should be turned on or off. The optimal policy will result in the lowest operating cost and highest efficiency of pump station [1].

Optimal pump scheduling policies will indeed decrease economic consumption of the whole flow systems. But, the dynamical and mixed-integer nature associated to the optimization of scheduling pump stations increases the complexity of the optimal control problem of water networks [2]. Some typical mixed-integer linear programming or dynamic programming-based algorithms are not applicable because of high computation load, being infeasible or may be not being efficient enough for large water networks [2][3].

MPC is a well-established class of advanced control methods [4][5] and is used for the control of distribution water networks [6][7] as it can compute advanced control strategies (e.g. to minimize energy cost) while guaranteeing certain quality of service and satisfying the operating constraints of the plant. MPC can handle large scale systems with operational constraints in states and inputs, multiple objectives and is flexible to extensions. However, the inclusion of discrete ON-OFF operation of pumps would lead to an hybrid

*This work has been funded by the Spanish Ministry of Science and Technology through the project CYCYT WATMAN DPI2009-13744 and also funded by EFFINET grant FP7-ICT-2012-318556 of the European Commission.

${ }^{1}$ Cong Cong Sun, Vicenç Puig and Gabriela Cembrano are with Advanced Control Systems Group at the Institut de Robòtica i Informàtica Industrial (CSIC-UPC), Llorens i Artigas, 4-6, 08028 Barcelona, Spain. e-mail:vicenc . puig@upc . edu

${ }^{2}$ Gabriela Cembrano is with CETaqua, Water Technology Centre at Esplugues 75, 08940, Cornellà de Llobregat, Barcelona e-mail:cembrano@iri.upc.edu
MPC problem that would probably involve computational problems in case of large scale water networks.

The main contribution of this paper is to propose a twolayer control scheme for optimizing the scheduling of pump stations, avoiding mixed-integer optimization problems. At the upper layer, MPC is used to optimize the continuous flow model and then, at the lower layer, scheduling algorithm translates the continuous flow set-points into ONOFF control operation sequences of the pumps. MATLAB and EPANET have been used to simulate and validate the proposed approach in the Richmond network case study [8].

This paper is organized as follows. Section II provides the control oriented modelling methodology for water distribution systems. Section III presents the MPC mixed-integer optimization problem for the scheduling of pump stations. Section IV provides the two-layer control scheme and describes the two layers individually. Section V provides the scheduling algorithm details with its tuning parameters. In Section VI, complexity analysis of this algorithm is provided. Then, in Section VII, the considered case study (Richmond Water Network) and the application results of the proposed methods are described and finally, the main conclusions are presented in Section VIII.

\section{CONTROL-ORIENTED MODELLING METHODOLOGY}

A control-oriented modelling approach is outlined for the upper layer, which follows the principles presented in [9], [7] and [10]. The extension to include the pressure-model can be found in references provided by [2] and [11].

\section{A. Tanks and Reservoirs.}

Water tanks and reservoirs provide the entire network with the water storage capacity. The mass balance expression of these storage elements can be written as the discrete-time difference equation

$$
v_{i}(k+1)=v_{i}(k)+\Delta t\left(\sum_{j} q_{u_{j}}(k)-\sum_{l} q_{u_{l}}(k)\right),
$$

where $v$ is the stored volume, $q_{u_{j}}$ are the manipulated inflows and $q_{u_{l}}$ are the manipulated outflows for the $i^{\text {th }}$ storage element, $\Delta t$ is the sampling time and $k$ denotes the discretetime instant. The physical constraint related to the range of admissible water in the $i^{\text {th }}$ storage element is expressed as

$$
\underline{v}_{i} \leq v_{i}(k) \leq \bar{v}_{i}, \quad \text { for all } k,
$$

where $\underline{v}_{i}$ and $\bar{v}_{i}$ denote the minimum and the maximum admissible storage capacity, respectively. 


\section{B. Actuators}

Two types of control actuators are considered: valves and pumps (more precisely, complex pumping stations). The manipulated flows through the actuators represent the manipulated variables, denoted as $q_{u}$. Both pumps and valves have lower and upper physical limits as system constraints. In (2), they are expressed as bound constraints

$$
\underline{q u}_{i} \leq q_{u_{i}}(k) \leq \bar{q}_{u_{i}}, \quad \text { for all } k,
$$

where ${\underline{q_{u}}}_{i}$ and $\overline{q_{u}}$ denote the minimum and the maximum flow capacity, respectively.

\section{Nodes.}

The nodes represent mass balance relations of inflows and outflows (e.g., because of a pipe junction).

$$
\sum_{j} q_{u_{j}}(k)=\sum_{h} q_{u_{h}}(k) .
$$

We define an incidence matrix $\Lambda_{c}$ for junction nodes in order to write equation (4) in matrix form, where the element in the $i^{\text {th }}$ column and $j^{\text {th }}$ row of junction nodes incidence matrix $\Lambda_{c}$ is defined as:

$$
a_{i j}= \begin{cases}1 & \text { if flow of branch } i \text { enters node } j \\ 0 & \text { if branch } i \text { and node } j \text { are not connected } \\ -1 & \text { if flow of branch } i \text { leaves node } j\end{cases}
$$

Assuming one network has $n_{c}$ static nodes and $n_{b}$ branches, thus, this matrix has $n_{c}$ rows and $n_{b}$ columns.

A matrix form of equation (4) is as follows:

$$
\Lambda_{c} q_{u}(k)=d(k)
$$

where $q_{u}=\left(q_{u_{1}}, \ldots, q_{u_{n_{b}}}\right)^{T}$ is a vector of branch flows, $d$ denotes an augmented demand vector where zero components to nodes without demand associated with.

\section{Demand Sectors.}

Demand and irrigation sector represents the water demand made by the network users of a certain physical area. The demand forecasting algorithm, that runs in parallel with the control algorithm, typically uses a two-level scheme composed by (i) a time-series model to represent the daily aggregate flow values, and (ii) a set of different daily flow demand patterns according to the day type to cater for different consumption during the weekends and holidays periods as provided by [12].

\section{E. Network control oriented model}

Finally, using the models of the different elements of the water network presented above, the following linear discretetime control oriented model can be obtained taking into account the network topology and parameters:

$$
\begin{aligned}
x(k+1) & =A x(k)+B u(k) \\
y(k) & =C x(k) \\
\Lambda_{c} u(k) & =d(k)
\end{aligned}
$$

where $x(k) \in \mathbb{R}^{n_{x}}$ is state vector that corresponds with the tank volumes and $u(k) \in \mathbb{R}^{n_{u}}$ is vector of command variables that correspond with the valve/pump flow set-points, while $y(k) \in \mathbb{R}^{n_{y}}$ is vector of the measured output that correspond with the tank levels.

\section{PROBLEM PRESENTATION}

Let us consider that the pump scheduling time horizon $\left[t_{0}, t_{f}\right]$ can be split into $K$ time steps with $\Delta t_{k}$ length each, where $\Delta t_{k}=t_{k}-t_{k-1} ; k=1, \ldots, K, t_{f}=t_{k}$. This results in timing of the scheduling problem which is determined by the intervention time instants $t_{0}, t_{1}, \ldots, t_{k}, \ldots, t_{f}$. Naturally, the system control vector, $p(k)$, represents status of pumps $(\mathrm{ON}-$ OFF) in each of these time stages [2].

The pump scheduling problem (PSP) for a given time horizon can be formulated as follows:

$$
\begin{array}{ll} 
& \min _{p, \Delta t_{k}} \sum_{k=1}^{K} \alpha(k) \tilde{u}(k) p(k) \Delta t_{k} \\
\text { s.t. } & x(k)=A x(k)+B \tilde{u}(k), \quad k=1, \cdots, K \\
& x(0)=x_{0}, \quad k=1, \cdots, K \\
& \Lambda_{c} \tilde{u}(k)=d(k) \\
& x_{\text {min }} \leq x(k) \leq x_{\text {max }}, \quad k=1, \cdots, K \\
& \tilde{u}_{\text {min }} \leq \tilde{u}(k) \leq \tilde{u}_{\text {max }}, \quad k=1, \cdots, K \\
& p(k) \in\{0,1\}, \quad k=1, \cdots, K
\end{array}
$$

where $\tilde{u}(k)$ is the nominal pump flows (when the pump is $\mathrm{ON})$ and $\alpha(k)$ is the unitary electrical costs for the $k$ time stage, $x(k)$ represents the continuous tank volumes, and the system operating cost associated to pumping.

The PSP is solved by selecting a proper $\Delta t_{k}$ according to pump operatinal constraints and pump control sequence $p$ that requires minimal economic pumping cost while satisfying flow or volume requirements induced by the demands. The small value of $\Delta t_{k}$ and the complex topology and number pumps could consequently increase the computation load. The mixture of discrete control parameters (ON-OFF pump schedule) together with the continuous dynamics of tank volumes makes PSP problem a complex mixed-integer optimization problem [2].

For this complex mixed-integer problem, the method of conversion of the mixed-integer problem into the continuous one by switching times as the control variables is indeed useful, but the solution is obtained at the expense of an increased number of decision variables, whose applicability is limited to rather small networks due to poor robustness with respect to numerical errors [2]. This paper will derive an efficient alternative approach which separates the mixedinteger problem into individual continuous and discrete problems by decomposition of the scheduling problem into a twolayer control scheme, as Fig. 1 shows.

\section{PRESENTATION OF THE TWO-LAYER CONTROL SCHEME}

As shown in Fig. 2, the proposed control scheme includes two layers. The upper layer is the continuous MPC model 


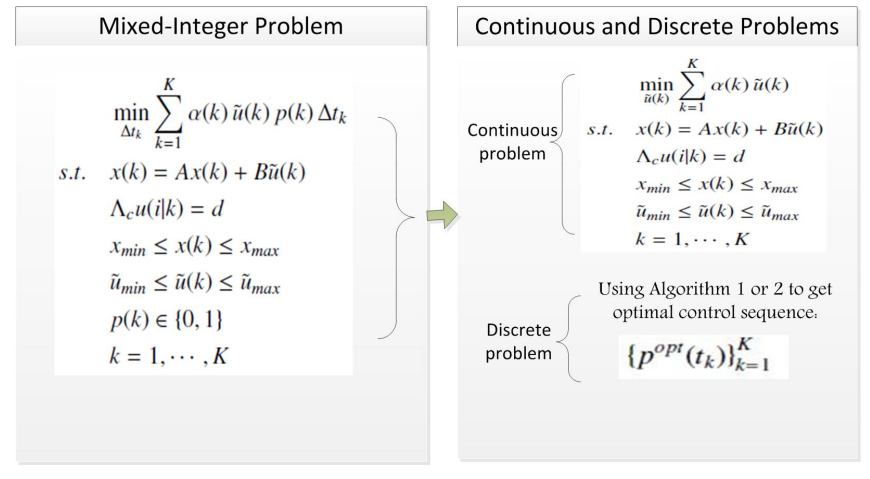

Fig. 1: Presentation of the proposed approach

that produces continuous pump flow set-points. Sampling time in the upper layer is one hour and every pump station is simplified into a controlled flow $u(k)$ and cost (electricity price) model $\alpha(k)$. The lower layer is the scheduling algorithm, who works in $\Delta t_{k}$ (smaller than one hour) sampling time, is responsible of translating the continuous flow setpoints into discrete ON-OFF actions to be executed by the pumps. The resulting pump schedule is simulated by EPANET before being sent to real pumps in the network.

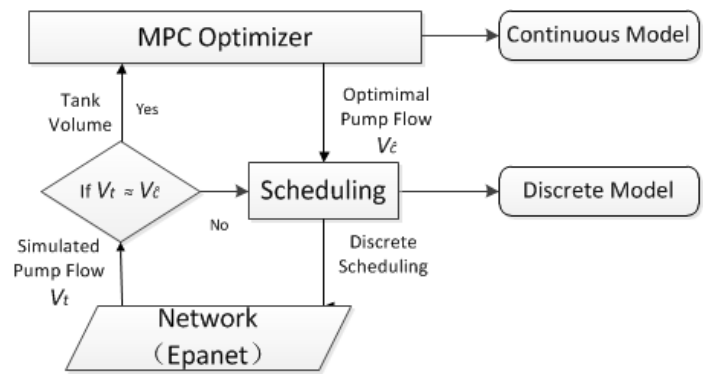

Fig. 2: Two-layer Control Scheme

\section{A. Optimizing Flow at the Upper layer}

MPC is used to produce optimal continuous set-points pump flows for being scheduled in the lower layer. The extension to include non-linear pressure model is researched in parallel in [13], which uses CSP (Constraints Satisfaction Problem) to transfer the non-linear MPC into linear ones with added constraints.

The upper layer MPC problem is based on a linear discrete-time prediction model obtained applying the control oriented methodology introduced in Section II considering the network topology and parameters:

$$
\begin{array}{ll} 
& \min _{\left(u(0 \mid k), \cdots, u\left(H_{p-1} \mid k\right)\right)} J(k) \\
\text { s.t. } & x(i+1 \mid k)=A x(i \mid k)+B u(i \mid k), \quad i=1, \cdots, H_{p} \\
& x(0 \mid k)=x_{k} \\
& \Lambda_{c} u(i \mid k)=d \\
& x_{\min } \leq x(i \mid k) \leq x_{\max }, \quad i=1, \cdots, H_{p} \\
& u_{\min } \leq u(i \mid k) \leq u_{\max }, \quad i=0, \cdots, H_{p-1}
\end{array}
$$

As described above $J$ is a performance index, representing operational goals of the system, $H_{p}$ is the prediction horizon, $x(0)$ is the initial condition of the state vector, $x_{\min }$ and $x_{\max }$ are minimal and maximal capacities of tanks, while $u_{\min }$ and $u_{\max }$ are known vectors defining the saturation constraints on inputs variables. Problem (9a) can be recast as a Quadratic Programming (QP) problem, whose solution:

$$
\mathcal{U}^{*}(k) \triangleq\left[u^{*}(0 \mid k) \cdots u^{*}\left(H_{p}-1 \mid k\right)\right]^{T} \in \mathbb{R}^{H_{p} m \times 1}
$$

is a sequence of optimal control inputs that generates an admissible state sequence. Only the first optimal move $u^{*}(0 \mid k)$ of the optimal sequence $\mathcal{U}^{*}(k)$ is applied to the optimal process and the optimization is repeated at time $k+1$.

1) Operational Goals: The water distribution network is operated with a 24-hour horizon, at hourly time interval. The main operational goals to be achieved are:

- Cost reduction $\left(J_{\text {cost }}\right)$ : Minimize water cost during water supplying process, which includes operating cost of the pump station and also water source cost.

- Operational safety $\left(J_{\text {safety }}\right)$ : Maintain appropriate water storage levels in dams and reservoirs of the network for emergency-handling situations.

- Control actions smoothness ( $\left.J_{\text {smoothness }}\right)$ : Smooth flow set-point variations for sustainable process operation.

Above mentioned goals lead to the following function:

$$
\begin{aligned}
J & =J_{\text {safety }}+J_{\text {smothness }}+J_{\text {cost }} \\
& =\varepsilon_{\widetilde{x}}(k)^{\top} W_{\widetilde{x}} \varepsilon_{\widetilde{x}}(k)+\Delta \widetilde{u}(k)^{\top} W_{\widetilde{u}} \Delta \widetilde{u}(k) \\
& +W_{a}\left(a_{1}+a_{2}(k)\right) \widetilde{u}(k)
\end{aligned}
$$

where

$$
\begin{aligned}
\varepsilon_{\widetilde{x}}(k) & =\widetilde{x}(k)-\widetilde{x}_{r} \\
\widetilde{u} & =\Theta \Delta \widetilde{u}+\Pi \widetilde{u}(k-1) \\
\Delta \widetilde{u}(k) & =\widetilde{u}(k)-\widetilde{u}(k-1)
\end{aligned}
$$

$$
\Theta=\left(\begin{array}{cccc}
I_{m_{i}} & 0 & \ldots & 0 \\
I_{m_{i}} & I_{m_{i}} & \ldots & 0 \\
\vdots & \vdots & \ddots & \vdots \\
I_{m_{i}} & I_{m_{i}} & \ldots & I_{m_{i}}
\end{array}\right), \quad \Pi=\left(\begin{array}{c}
I_{m_{i}} \\
I_{m_{i}} \\
\vdots \\
I_{m_{i}}
\end{array}\right)
$$

and $W_{\widetilde{x}}, W_{\widetilde{u}}, W_{a}$ are also related weights.

The vectors $a_{1}$ and $a_{2}$ contain the cost of water treatment and pumping price, respectively and $\widetilde{x}_{r}$ is the penalty level for emergency-handling in reservoirs of the network.

2) Formulation of the optimization problem: The objective functions (11) of the MPC problem can be formulated in the following way:

$$
J=z^{T} \Phi z+\phi^{T} z+c
$$

where

$$
z=\left[\begin{array}{lll}
\Delta \widetilde{u} & \varepsilon_{\widetilde{x}} & \varepsilon
\end{array}\right]^{T}
$$

and $c$ is a constant value. 
This allows to determine optimal control actions at each instant $k$ by solving a quadratic optimization problem by means of quadratic programming (QP) algorithm in form as:

$$
\begin{gathered}
\min _{z} z^{\top} \Phi x+\phi^{\top} z \\
A_{1} z \leq b_{1} \\
A_{2} z=b_{2}
\end{gathered}
$$

\section{B. Pump scheduling of the Lower layer}

Denoting $\hat{c}$ as the optimal flow set-points produced by the upper layer MPC controller during the time period $\left[t_{0}, t_{f}\right]$, the total water volume pumped during this period is

$$
V_{\hat{c}}=\hat{c}\left(t_{f}-t_{0}\right)
$$

As explained in Section II, the scheduling algorithm will split the time period $\left[t_{0}, t_{f}\right]$ into $K$ time steps with $\Delta t_{k}$ step length. Let us denote $p$ as the vector which contains the discrete ON $(p(i)=1)$ and OFF $(p(i)=0)$ pump control actions [14] and $\tilde{u}(t)$ as its nominal pump flow (that is when the pump is $\mathrm{ON}$ ). Then, the total water volume drawn by these pump control actions during $\left[t_{0}, t_{f}\right]$ is

$$
V_{t}(p(1), \cdots, p(K))=\sum_{i=1}^{K} p(i) \int_{t_{i-1}}^{t_{i}} \tilde{u}(t) d t
$$

The goal of the scheduling algorithm is to minimize the difference between $V_{\hat{c}}$ and $V_{t}$ in (14) and (15). Since this difference could not completely be eliminated, the scheduling algorithm should find a scheduling sequence such that the following control objective is minimized

$$
J_{d i s}=V_{t}-V_{\hat{c}}
$$

\section{FACTORS AFFECT SCHEDULING ALGORITHMS}

There are two parameters which can affect accuracy of the scheduling algorithm as described in previous section

- time interval $\left(\Delta t_{k}\right)$

- number of units in the pump configuration

\section{A. Time interval}

In order to guarantee that the pump station configuration can meet the pump flow set-points provided by the upper layer, the sampling time can be selected to reduce the error introduced in (16).

Assuming $\Delta t_{k}$ is small enough in order to accurately compute the term $\int_{t_{i-1}}^{t_{i}} \tilde{u}(t) d t$ in (15) as $u^{*}(k) \Delta t_{k}$, where $u^{*}(k)$ is the nominal pump flow in time stage $\left[t_{k-1}, t_{k}\right]$, the equation (15) can be rewritten as:

$$
V_{t}(p(1), \cdots, p(K))=\sum_{i=1}^{K} p(i) \int_{t_{i-1}}^{t_{i}} \tilde{u}(t) d t \cong \sum_{i=1}^{K} p(i) u^{*}(k) \Delta t_{k}
$$

Consequently, the accuracy of scheduling algorithm according to (16) can be calculated as follows:

$$
J_{d i s}=\min \left(V_{t}-V_{\hat{c}}\right) \cong \min \left(\sum_{i=1}^{K} p(i) u^{*}(k) \Delta t_{i}-\hat{c}\left(t_{f}-t_{0}\right)\right)
$$

In practice, $J_{d i s}$ is affected by $\Delta t_{k}$, the smaller $\Delta t_{k}$ is, the smaller will be (18). However, the value of $\Delta t_{k}$ is limited by technological constraints imposed by the pumps. Pump scheduling algorithm with $\Delta t_{k}$ works as presented in Algorithm 1.

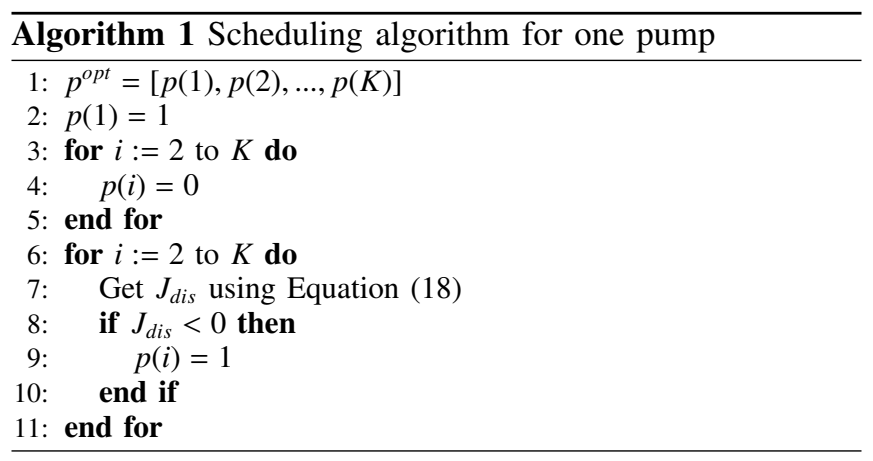

In this algorithm, $p^{o p t}$ is the optimal working schedule for the pump, and $J_{d i s}$ is the optimal scheduling accuracy.

\section{B. Parallel pump configuration}

If a single pump cannot meet the pump flow set-points determined in the upper layer, additional units in the pump station should be activated.

Assuming $n-1$ supplementary units are available at the pump station in order to minimize (16):

$$
V_{\hat{c}} \leq V_{1 t}+V_{2 t}+\ldots+V_{n}
$$

Then, scheduling accuracy of (18) could be evaluated as follows

$$
\begin{aligned}
J_{d i s} & =\min \left(V_{1 t}+V_{2 t}+\ldots+V_{n}-V_{\hat{c}}\right) \\
& \cong \min \left(\sum_{1}^{n} \sum_{i=1}^{K} p(i) u^{*}(k) \Delta t_{k}-\hat{c}\left(t_{f}-t_{0}\right)\right)
\end{aligned}
$$

where $n$ means the number of parallel units in the pump station which is another factor that could be used to increase the schedule accuracy: the bigger $n$ is, the more degrees of freedom and the higher accuracy could be achieved by means of the scheduling algorithm.

Algorithm 2 presents the extension of Algorithm 1 to $n$ parallel units of the pump station.

The values of $p_{n}{ }^{\text {opt }}$ are the optimal schedules of the parallel pumps, $J_{d i s}$ is the optimal scheduling accuracy.

\section{COMPLEXITY}

Regarding complexity, computation load of the scheduling Algorithm 1 is $K$, where $K=\frac{t_{f}-f_{0}}{\Delta t_{k}}$. This means that, $\Delta t_{k}$ can affect computation load of the algorithm since more computations will be added with a smaller $\Delta t_{k}$, and consequently decreased with a bigger $\Delta t_{k}$. The same reasoning can be used in case of Algorithm 2, where the computation load is $K^{n}$ and the computation load is increased when the number of units $n$ in parallel. Because of that, although smaller time interval and more parallel pumps can increase scheduling accuracy, more computation load is needed. Therefore, it is important to choose proper $\Delta t_{k}$ and $n$ even that establishes a trade-off between accuracy and computation load. 


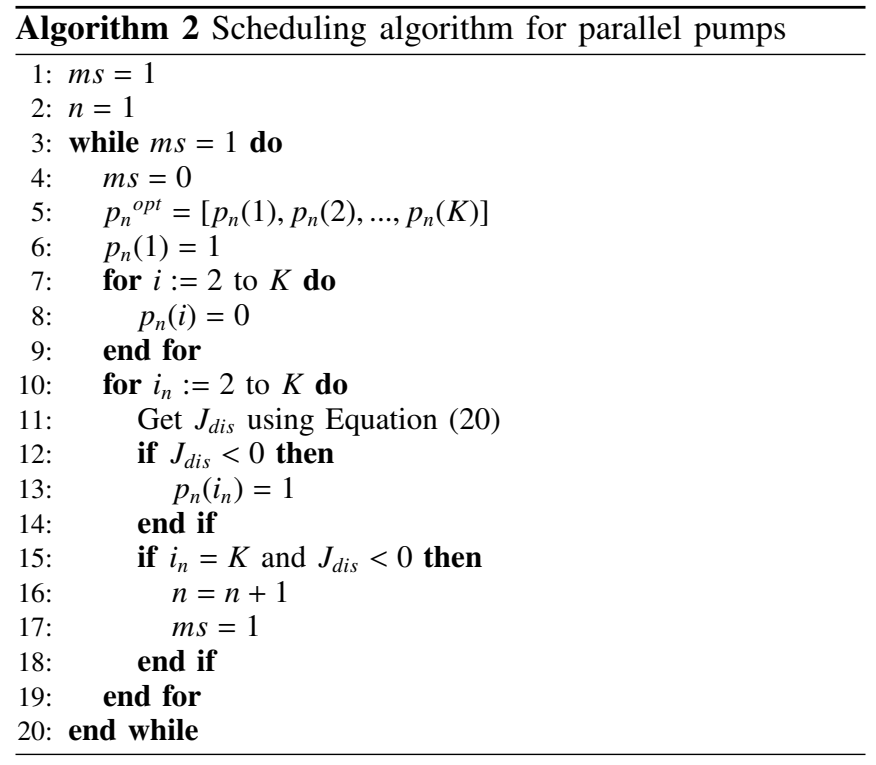

\section{CASE STUDY}

The case study used to test the proposed approaches is the Richmond water distribution system [15]. Fig. 3 shows the conceptual diagram of Richmond network. A MPC controller at the upper layer is used to produce the pump flow setpoints, while the pump scheduling algorithm described in Section IV-B is used to transfer the continuous flow setpoints into discrete $\mathrm{ON}-\mathrm{OFF}$ operations of the pump.

The MPC controller and the pump scheduling algorithm are implemented into MATLAB, while the simulation of the Richmond network is realized using EPANET, which simulates the water network using a discretization time step $\Delta t_{k}$ to realize operations of the scheduling algorithm.

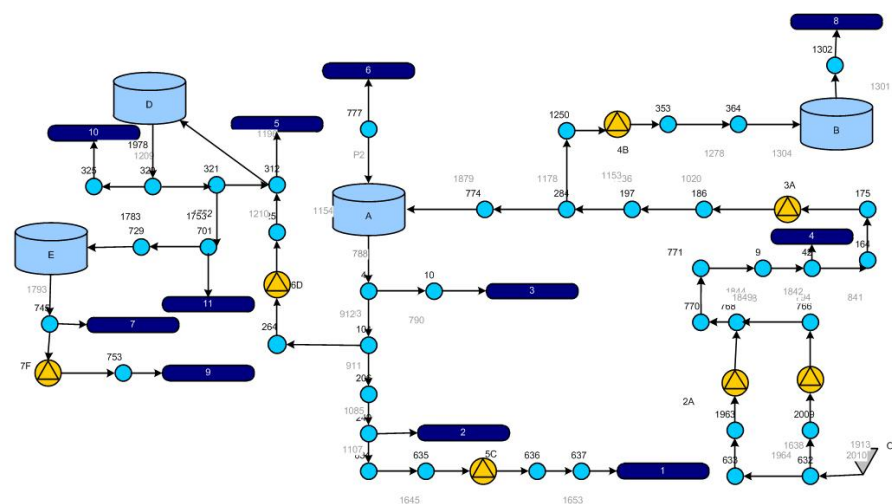

Fig. 3: The Richmond water distribution system

\section{A. Results for the upper layer MPC controller}

As described in Section IV-A, the objective function of the upper layer MPC controller leads to minimize the electrical pumping cost. Fig. 4 shows pump flow together with its electricity fee. From this figure, it is confirmed that pump send more water at its lower price period but less or no water at the higher price period, which is consistent with cost term in the objective function.

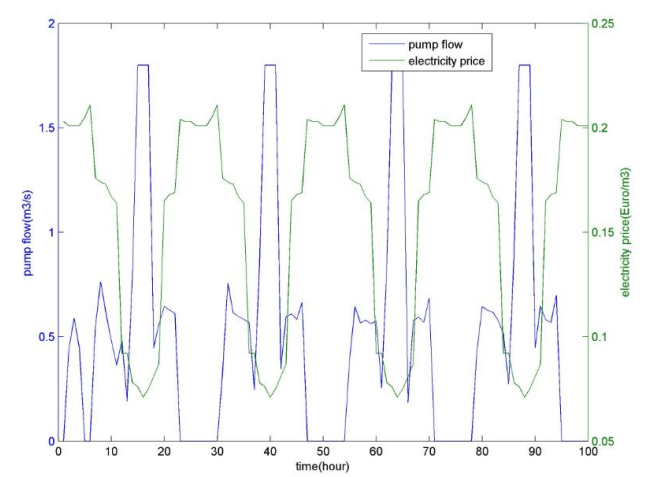

Fig. 4: Pump flow with electricity price

\section{B. Results for the lower layer scheduling algorithm}

After applying scheduling algorithm, continuous optimal flow will be scheduled into discrete pump actions. Figure 5 shows in detail the pump actions of Pump $4 B$ after using the scheduling algorithm.

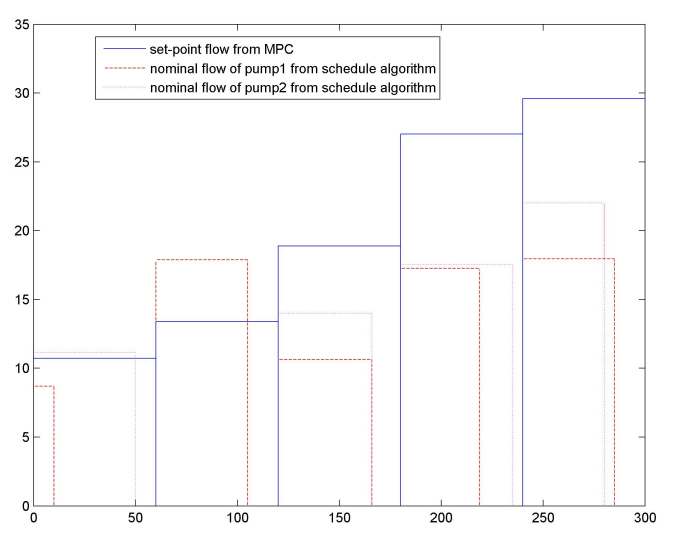

Fig. 5: Optimal Schedule for Pump4B with two pump branches

\section{Scheduling Results using Different $\Delta t_{k}$}

As analyzed in Section IV, time interval $\Delta t_{k}$ can affect accuracy of scheduling algorithm. Take pump $4 B$ as an example. In this case, the sampling time at the upper layer that determines $t_{f}-t_{0}$ is equal with 1 hour while the sampling time at the lower layer will be changed from one minute to two minutes to see the effect in the scheduling algorithm result. $\Delta t_{k}$ will use 1 minute and 2 minute two different values. Scheduling accuracies for these two different sampling times at lower layer are plotted in Fig. 6, which proves that, the smaller time interval can lead to higher scheduling accuracy.

Accuracy comparisons are provided in detail in Table I, which shows that, the scheduling accuracy when lower layer sampling time $\Delta t_{k}$ is 1 minute results in $0.64 \%$, which is much smaller than that of $1.71 \%$ when $\Delta t_{k}$ is 2 minutes. 


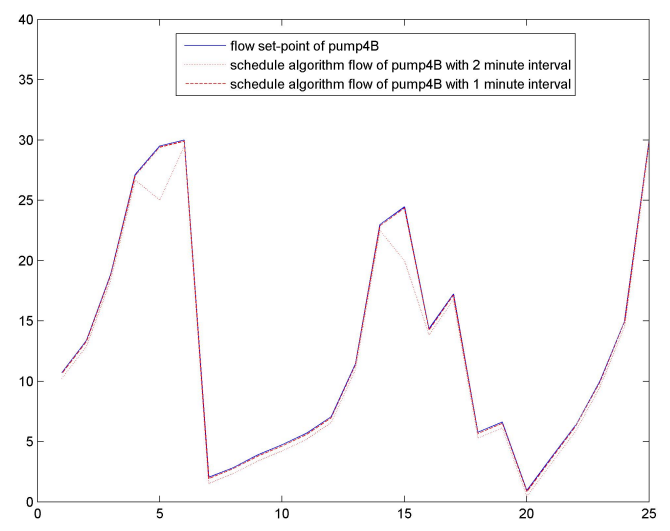

Fig. 6: Flow errors in different time intervals

TABLE I: Accuracy Comparison

\begin{tabular}{lllll}
\hline Sc. & \multicolumn{4}{c}{ With 2-minute Time Interval } \\
\hline Es. & Optimal flow & Simulated flow & Flow Errors & Errors in Prop. \\
\hline T. & 332.4045 & 326.7351 & 5.6694 & $1.71 \%$ \\
\hline Sc. & \multicolumn{4}{c}{ With 1-minute Time Interval } \\
\hline Es. & Optimal flow & Simulated flow & Flow Errors & Errors in Prop. \\
\hline T. & 329.6 & 327.4818 & 2.1182 & $0.64 \%$ \\
\hline
\end{tabular}

\section{Scheduling Results for Different Pump Configurations}

Number of parallel pump branches can also affect scheduling accuracy, since the bigger the number is, the higher the accuracy is. Considering $t_{f}-t_{0}$ of pump $4 B$ as 1 hour, $\Delta t_{k}$ as 1 minute cases with single pump $4 B$ and two paralleled pump pump $4 B$ are simulated. Their accuracies are provided in Fig. 7.

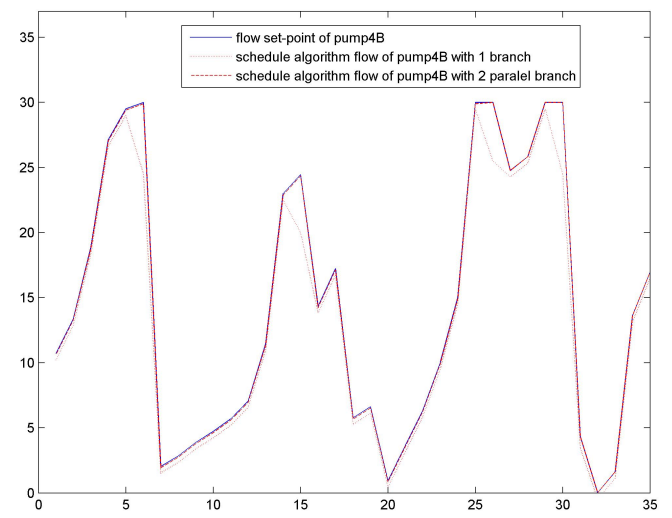

Fig. 7: Flow errors in different parallel when $\Delta t_{k}=1$

Accuracy comparisons are provided in detail in Table II, which shows that, the scheduling accuracy at the paralleled pump station is nearly $100 \%$ and much higher than that at the single pump.
TABLE II: Accuracy Comparison

\begin{tabular}{|c|c|c|c|c|}
\hline Sc. & \multicolumn{4}{|c|}{ With Single Pump } \\
\hline Es. & Optimal flow & Simulated flow & Flow Errors & Errors in Prop. \\
\hline T. & 526.7603 & 521.3726 & 5.3877 & $1.02 \%$ \\
\hline Sc. & \multicolumn{4}{|c|}{ With 2 Pumps in Parallel } \\
\hline Es. & Optimal flow & Simulated flow & Flow Errors & Errors in Prop. \\
\hline T. & 518.1651 & 518.0742 & 0.0909 & $0.01 \%$ \\
\hline
\end{tabular}

\section{CONCLUSION}

This paper has proposed a two-layer control scheme for scheduling pump stations in water distribution networks in order to solve the mixed-integer optimization problem. The upper layer, who works in one-hour sampling time, uses a MPC strategy to optimize continuous flow model to produce set-point pump flows for the lower layer. While in the lower layer, a scheduling algorithm is used to translate the optimal continuous set-points flow into ON-OFF pump operations. The effect of tuning parameters (sampling time and pump configurations) of the scheduling algorithm is compromised chose in reality according to scheduling accuracy and computation load.

\section{REFERENCES}

[1] L. E. Ormsbee and K. E. Lansey, "Optimal control of water supply pumping systems," Journal of Water Resour. Plann. Management, vol 120, p. 237252, 1994.

[2] M. Brdys and B. Ulanicki, Operational Control of Water Systems: Structures, algorithms and applications. UK: Prentice Hall International, 1994.

[3] B. Coulbeck and C. Orr, "Development of an interactive pump scheduling program for optimized control of bulk water supply," Proc. of Int. Cont. on Systems Engineering, Lanchester Polytechnic, pp. 17686, 1981.

[4] J. B. Rawlings and D. Q. Mayne, Model predictive control: theory and design. Madison: Nob Hill Publishing, 2009.

[5] D. Q. Mayne, J. B. Rawlings, C. V. Rao, and P. O. M. Scokaert, "Constrained model predictive control: stability and optimality," $\mathrm{Au}$ tomatica, vol. 36, pp. 789-814, 2000.

[6] J. Grosso, C. Ocampo-Martnez, and V. Puig, "Learning-based tuning of supervisory model predictive control for drinking water networks," Engineering Applications of Artficial Intelligence, 2013.

[7] C. Ocampo-Martinez, D. Barcelli, V. Puig, and A. Bemporad, "Hierarchical and decentralised model predictive control of drinking water networks: Application to barcelona case study," IET Control Theory and Applications, vol. 6(1), pp. 62-71, 2012.

[8] H. S. S. AbdelMeguid, "Pressure, leakage and energy management in water distribution systems," Ph.D. dissertation, De Montfort University, 2011.

[9] G. Cembrano, J. Quevedo, M. Salamero, V. Puig, J. Figueras, and J. Martí, "Optimal control of urban drainage systems: a case study," Control Engineering Practice, vol. 12, no. 1, pp. 1-9, 2004.

[10] C. Ocampo-Martinez, V. Puig, G. Cembrano, and J. Quevedo, "Application of predictive control strategies to the management of complex networks in the urban water cycle," IEEE Control Systems Magazine, vol. 33, no. 1, pp. 15-45, 2013

[11] L. Mays, Urban Stormwater Management Tools. USA: McGrawHill Professional Publishing, 2004.

[12] J. M. Maciejowski, Predictive Control with Constraints. Great Britain: Prentice Hall, 2002.

[13] V. P. C. C. Sun and G. Cembrano, "Combining csp and mpc for the operational control of water networks: Application to the richmond case study," The 19th World Congress of the International Federation of Automatic Control, 2014.

[14] B. U. M.A. Brdys, Operational Control of Water System. London: Prentice Hall, 1994.

[15] U. of Exeter, "Center for Water Systems," University of the year 2012 2013, 2012, http://emps.exeter.ac.uk/engineering/research/cws /resources/benchmarks/operation/richmond.html. 